\title{
The Effect of Brandt-Daroff Exercise on the Vestibular Organ of Women with Vertigo
}

\author{
Dongwook Han, PhD, PT ${ }^{1)}$, Chanmi Yang, $\mathrm{PT}^{1)}$, Hyemin Yu, $\mathrm{PT}^{1)}$, \\ Misook Ha, PhD, $\mathrm{PT}^{2}$, Youngmin Son, $\mathrm{PT}^{3)}$ \\ 1) Department of Physical Therapy, College of Medical and Life Science, Silla University \\ 2) Department of Physical Therapy, ChoonHae College of Health Sciences: Ungchon-myeon, Ulju-gun, \\ Ulsan,689-784 Korea.TEL:+82 52-270-0342,FAX:+82 52-270-0199,E-mail:msha@ch.ac.kr \\ 3) Department of Physical Therapy, Daedong Hospital
}

\begin{abstract}
Purpose] The purpose of this study was to find the effects of the Brandt-Daroff exercise on vestibular functions, such as balance, perception of subjective visual vertical (SVV), and nystagmus, in women with vertigo. [Subjects] After asking a group of women five questions about vertigo symptoms, we chose 17 women, in the age range of 19 to 21, who each had more than one symptom to participate in this study. [Methods] Vestibular functions were assessed through the use of a balance pad, subjective visual vertical (SVV), and videonystagmography (VNG). Each subject performed the Brandt-Daroff exercise five times a day for two weeks from May 8th to May 21th in 2010. Afterwards, vestibular functions were assessed by the same measuring instruments. After the intervention, the changes in vestibular functions were analyzed by the Wilcoxon sign rank test and repeated measures ANOVA test with the SPSSWIN (ver 18.0) package program. [Results] After the Brandt-Daroff exercise, perception of SVV in the left eye and right eye improved. In VNG, involuntary eye movement improved after exercise. However, neither measured equilibrium on the balance pad nor SVV in both eyes had changed. [Conclusion] This study revealed that two weeks performance of the Brandt-Daroff exercise improves some vestibular functions of women with vertigo. However, some functions did not change. Therefore, it seems that another study is needed to assess the effects of exercise for more than two weeks.
\end{abstract}

Key words: Brandt-Daroff exercise, Vertigo, Vestibular organ

(This article was submitted Dec. 12, 2011, and was accepted Jan. 17, 2012)

\section{INTRODUCTION}

Vertigo appears with various other symptoms when there is an orientation disorder, and inability to hold balance generally occurs when there is imbalance among the vestibular system, visual system and proprioceptive system.

Vertigo can be classified into two types, one is central and the other is peripheral, according to the problematic part of the scala vestibuli. When the inner ear, which senses, has a disorder, this is called peripheral vertigo, whereas when the brainstem and cerebellum, which process information, have a problem, it is called central vertigo $\$$. Vertigo caused by peripheral scala vestibuli tends to occur intermittently and this type of vertigo is made worse by perceived movement of the circumjacent environment and movement of the head. Furthermore, paracusis or neurologic abnormalities are often symptoms of abnormality in the peripheral scala vestibuli. Those who have central scala vestibuli report that they have persistent vertigo, perceiving a spinning sensation when stationary, and that symptoms get better when they move their head . In addition, scala vestibuli vertigo can be suspected when syncope is a factor 7 .

Vertigo is generally treated by performing surgery as a repositioning maneuver and administering such drugs as antihistamines and anticholinergics. Long-term drug use, however, can cause inhibition of the central adaptive mechanism, leading to hindrance of the compensatory mechanism and slowing the rate of recovery. Hence, prompt vestibular rehabilitation must be performed as soon as possible after the acute stage has passed so as to stimulate

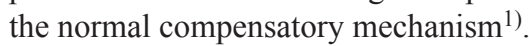

Although vestibular rehabilitation is pivotal to vertigo treatment, the study of vertigo has been most frequently been conducted in the field of otolaryngology so far, and studies of the effect of physical therapy on vertigo are rare. Although many studies of the clinical analysis, the effects of on vertigo have been published, there is only one case study of the effect of exercise on surgery, and drug treatment vestibular rehabilitation. Therefore, there is a need for studies of methods of rehabilitation that involve stimulating the compensatory mechanism of the scala vestibuli without the side effects of surgery or drugs. Accordingly, this study examined if fortifying vestibular functions using BrandtDaroff exercise is effective in vertigo treatment. 


\section{SUBJECTS AND METHODS}

In this study, 17 women who had more than one symptom of vertigo were chosen as subjects, through a medical examination interview, from among female university students living in Busan. The way of selecting the subjects was as follows. First, we selected subjects who reported they had dizziness in daily life. Then we selected those subjects who reported having more than one symptom assessed among five symptoms by questionnaire, and confirmed that they had nystagmus by videonystagmography (VNG). If a subject did not having nystagmus, she was excluded from the study. The subjects of this study had not been diagnosed with vertigo or treated for it. The average age of the selected women was $20.12 \pm 0.332$ years, their average height was 160.88 $\pm 4.09 \mathrm{~cm}$, and their average weight was $53.59 \pm 6.0 \mathrm{~kg}$. Before proceeding with the experiment, all subjects were informed about the experiment and its procedure, and they agreed to participate by signing a document of consent. The medical interview evaluated the 5 factors below:

1. feeling dizziness when waking up $\$$.

2. feeling dizziness when nodding one's head up or down 9 .

3. feeling dizziness when lying down, getting out of bed or turning around in bed $\$$.

4. feeling dizziness when getting in a car or getting on a ship or flight p. $^{2}$.

5. feeling dizziness when looking down from a high place $\bar{f}$.

To diagnose vertigo, a subject's medical history is recorded, and a medical examination by interview is performed. A physical examination and neurological examination are also performed in order to observe nystagmus, and either oculography is checked or a positioning test is done 7 . This study used a balance pad test, the subjective visual vertical (SVV) test, and the videonystagmography (VNG) test in order to diagnose vertigo. The examiner had over 15 years of testing vertigo using VNG.

The balance pad test was performed as follows. With the subjects' two eyes covered by eye-patches, they were askred to shake the head from left to right ten times while keeping the body motionless. After the vestibular function was stimulated, subjects' were asked to step on a resilient balance pad, which prevents proprioception of the subject, on two feet so as to count how many seconds they could stand on it. Through this method only vestibular functions were measured.

The Subjective Visual Vertical (SVV) test is performed as follows. The otolith organ plays a central role in sensory input for maintaining normal posture and balance. The subjective visual vertical is typically used to evaluate the function of this organ $\$$. To take the measurement, a chair is positioned $1.5 \mathrm{~m}$ away from a monitor in a silent dark room. When measurements are taken, a pole or stick is set up and randomly positioned on the monitor by a software program. Subjects underwent this test while sitting on a chair, with the center of the pole or stick situated in the front field of vision.

The videonystagmography (VNG) test is performed as follows. This test involves capturing the image of an eyeball
Table 1. Change of balance pad $(\mathrm{N}=17)$

\begin{tabular}{lll}
\hline Variation & Pre-exercise & Post-exercise \\
\hline Balance pad & $1.26 \pm 0.59$ & $1.70 \pm 0.81$ \\
\hline Mean \pm SD & &
\end{tabular}

by infrared CCD camera to determine the spot of the pupil and the pattern of the iris, and the horizontal and vertical nystagmus can be analyzed accurately based on variation of pupil location. In this study, the existence of nystagmus and its severity were analyzed by spontaneous nystagmus, Dix-Hallpike right, Dix-Hallpike left, positional head, head hanging, and head shaking.

The Brandt-Daroff exercise method is as follows. [Stage 1] Sit upright for 30 seconds. [Stage 2] Lie on one side while looking up at an angle of 45 degrees for 30 seconds. [Stage 3] Sit upright for 30 seconds again. [Stage 4] Lie on the other side while looking up at an angle of 45 degrees for 30 seconds. [Stage 5] Finish the exercise by sitting upright for 30 seconds. Participants were asked to repeat the exercise, doing more than 5 sets (one set consisted of stages 1-5) a day over a 2 -week period. Participants were cautioned not to repeat the 5 sets consecutively, but periodically, so that its effect would not be encumbering.

To analyze the outcome of the intervention as well as to see the effect of the Brandt-Daroff exercise on improvement of subjects' vestibular functions, the variation of vestibular functions before and after the exercise was analyzed using non-parametric statistics, the Wilcoxon sign rank test. The data was processed using the software package SPSSWIN (ver. 18.0) and was accepted for values of statistical significance $\alpha \leq 0.05$.

\section{RESULTS}

The effect of Brandt-Daroff exercise on balance function had was no significant effect on the balance pad test $(\mathrm{p}>0.05)$ (Table 1).

In SVV, there were significant differences between measurements before and after the Brandt-Daroff exercise on the Left $(\mathrm{p}<0.05)$ and Right $(\mathrm{p}<0.05)$ but there was no significant difference in Both ( $p>0.05)$ (Table 2). After doing the exercise, the occurrence of nystagmus diminished, and the VNG test $(\mathrm{p}<0.05)$ showed significant improvements (Table 3).

\section{DISCUSSION}

Vertigo can be accompanied by balance disorder, vomiting, syncope and other troubles. It can also be caused by an abnormality of the scala vestibuli. In modern times, drugs and surgery are relied upon as solutions for remedying vertigo, and many studies of vertigo have been carried out with great interest in the discipline of otorhinolaryngology. However, there are relatively few methods to treat vertigo and improve scala vestibuli in terms of exercise or physical therapy approaches. For this reason, this study investigated the therapeutic effect of the Brandt-Daroff exercise, which 
Table 2. Changes of Subjective Visual Vertical (SVV) $(\mathrm{N}=17)$

\begin{tabular}{lll}
\hline Variation & Pre-exercise & Post-exercise \\
\hline Both & $1.30 \pm 0.61$ & $1.03 \pm 0.37$ \\
Left* & $1.29 \pm 0.48$ & $0.93 \pm 0.41$ \\
Right* & $1.51 \pm 0.66$ & $1.02 \pm 0.33$ \\
\hline
\end{tabular}

Mean \pm SD, * : $p<0.05$

Table 3. Change of Videonystagmography (VNG) $(\mathrm{N}=17)$

\begin{tabular}{lll}
\hline Variation & Pre-exercise & Post-exercise \\
\hline Nystagmus* & $1.00 \pm 0.000$ & $1.35 \pm 0.493$ \\
\hline Mean $\pm \mathrm{SD}, *: \mathrm{p}<0.05$ &
\end{tabular}

was designed to treat vertigo and improve the function of the scala vestibuli.

The Brandt-Daroff exercise was initially developed to cure patients who suffer from BPPV (benign paroxysmal positional vertigo) caused by posterior canal ampullary crest lithiasis 10 . Although the Brandt-Daroff exercise was designed as habitual physical therapy with repetitive movement, rather than as a redressment for detaching the otolith, it has the effect of detaching otoliths that have become attached through ampullary crest lithiasis and is useful in the treatment of veritigd ${ }^{11}$. Cheryl reported a case study in 1997 of a 32 year-old male patient diagnosed with BPPV, who had suffered from vertigo and severe headaches for 17 years, who performed the Brandt-Daroff exercise and eye-movement exercise. The eye-movement and Brandt-Daroff exercises were done twice a day at home. Although vertigo symptoms did not diminish after repeating the exercise for 24 days, the symptoms disappeared after 1 year on the exercise program. Cheryl reported that the eye movement exercise and the Brandt-Daroff exercise cured vertigo, but only over the course of time. Cheryl's study ${ }^{12}$, however, combined the Brandt-Daroff exercise with other treatments so that the effect of the Brandt-Daroff exercise could not be isolated. Therefore, this study was designed to examine, if the Brandt-Daroff exercise has an effect on the vestibular functions of people suffering general vertigo symptoms, not solely those of patients being treated for vertigo.

When subjects of this study did the Brandt-Daroff exercise 5 times a day for two weeks, the balance pad test showed no significant difference in the balance, but it was ascertained that the discrepancy between left and right decreased in the SVV test. In addition, the nystagmus of subjects diminished in the VNG test. Consequently, we have confirmed that Brandt-Daroff exercise has a positive effect on vestibular functions. This result is in agreement with that of the study of Horak and others ${ }^{13}$. In that study, they took patients with benign paroxymal positional vertigo (BPPV), which is a common cause of dizziness, as subjects and compared the effects of vestibular rehabilitation, general conditioning exercise, and vestibular suppressant medication. They reported that vestibular rehabilitation was more effective than other methods. Furthermore, Cohen et al ${ }^{14}$. reported that the patients with BPPV who were treated with the canalith repositioning maneuver (CRP), a modified Brandt-Daroff exercise, showed improvements. The Brandt-Daroff method uses repetitive motion to loosen debris that are attached to the ampulla and the arch falls produces a dramatic effect lymph dispersing. Inside the ring late in the debris no longer be distributed as a lump swollen less biased because it does not move will be. In the present study, the Brandt-Daroff method of moving the head, eyes, and body to stimulate the scala vestibuli proved to be effective for improvement of the vestibular functions. Nevertheless, we must take into consideration that the period of this study's exercise program was limited to 2 weeks - a relatively short period to confirm its effectiveness. Also, since our subjects were women in their 20s who suffered dizziness but had not been diagnosed with vertigo, the results of this study cannot be generalized to all ages, genders, and patients. Accordingly, we consider necessary to perform more syudies making allowances for gender and age, as well as including patients as subjects.

There are relatively few studies of physical therapy approaches stimulating the scala vestibuli compared to the extensive research that has been done in the field of balance and otorhinolaryngology. Hence, this study is significant in that it is a new approach in Korea. We hope our study will pique the interest of physical therapists, and provide useful data on vertigo and improvement exercises for the scala vestibuli.

\section{REFERENCES}

1) Lee KK: What can be old age patients with vertigo. J of the Korean Academty of Clinical Geriatrics. Spring Conference, 2006.

2) Moon WS, Jeong KU, Wee JS, et al.: Clinical analysis of the origins of dizziness in patients. J Korean Soc Emerg Med, 2001, 12: 259-267.

3) Hotson JR, Baloh RW: Acute vestibular syndrome. N Engl J Med, 1998, 339: 680-685. Medline [CrossRef

4) Cho YJ: Diagnostic approach to the dizzy patient. Inje Univ Med J, 2002, 4: $245-251$.

5) Lopez Escamez JA, Gamiz MJ, Fernandez Perez A, et al.: Impact of treatment on health-related quality of life in patients with posterior canal benign paroxysmal positional vertigo. Otol Neurotol, 2003, 24: 637-641. Medline CrossRef

6) Baloh RW, Honrubia V, Jacobson K: Benign positional vertigo: clinical and oculographic features in 240 cases. Neurology, 1987, 37: 371-378. Medline

7) Hilton M, Pinder D: The Epley manoeuvre for benign paroxysmal positional vertigo- a systematic review. Clin Otolaryngol Allied Sci, 2002, 27: 440-445. [Medline] CrossRef

8) Rine RM, Schubert MC, Balkany TJ: Visual-vestibular habituation and balance training for motion sickness. Phys Ther, 1999, 79: 949-957. Medline

9) Rhee CK, Park H: Evaluation of the otolithic function in unilateral peripheral vestibulopathy using ocular counter-rolling (OCR) and subjective visual horizontal(SVH). Korean J Otolaryngol, 1999, 42: 306-310.

10) Brandt $T$, Daroff RB: Physical therapy for benign paroxysmal positional vertigo. Arch Otolaryngol, 1980, 106: 484-485. [Medline] [CrossRef]

11) Lee SC: Respositioning maneuver of vertical canal benign paroxysmal positional vertigo. J Korean Balance Soc, 2005, 4: 333-338.

12) Ford-Smith CD: The individualized treatment of a patient with benign paroxysmal positional vertigo. Phys Ther, 1997, 77: 848-855. [Medline]

13) Horak FB, Johns-Rycewicz C, Black O, et al.: Effects of vestibular rehabilitation on dizziness and imbalance. Otolaryngol Head Neck Surg, 1992 106: 175-180. Medline

14) Cohen HS, Sangi-Haghpeykar H: Canalith repositioning Variations for benign paroxysmal positional vertigo. Otolaryngol Head Neck Surg, 2010, 143: 405-412. [Medline [CrossRef 